\title{
Du nouveau du côté de la Société internationale francophone d'éducation médicale (SIFEM)
}

\author{
Some news from the SIFEM
}

Le $6^{\mathrm{e}}$ Forum international francophone de pédagogie des sciences de la santé, qui s'est tenu à Montréal du 22 au 24 mai dernier, a été l'occasion pour notre société de faire le point sur nos actions en cours et de lancer de nouveaux groupes de travail ou de réflexion.

Les trois grands groupes d'action prioritaires portent respectivement sur la responsabilité sociale des facultés de médecine, la recherche en éducation médicale et la communication dans la pratique professionnelle en santé. Nous avons déjà évoqué le travail de ces groupes ${ }^{[1]}$ et certains résultats de leurs travaux ont déjà été publiés dans Pédagogie Médicale.

\section{Le groupe d'action « responsabilité sociale des facultés de médecine »}

La première phase de l'action du groupe « responsabilité sociale des facultés de médecine»a consisté à informer et sensibiliser les communautés des facultés, après avoir publié en langue française le texte de consensus mondial ${ }^{[2]}$. Au total, 1260 personnes ont répondu au questionnaire en ligne. La deuxième phase, qui vient de débuter en octobre, concerne l'expérimentation d'un projet de rechercheaction dans chacune des 29 facultés partenaires du projet. Cette expérimentation sera articulée autour d'une thématique choisie par la faculté pour une mise en œuvre concrète des principes de responsabilité sociale. Trois thématiques prioritaires ont été reconnues: contrat faculté-territoire, première ligne de santé et adaptation de la formation, mais d'autres thématiques pourront être aussi choisies par les facultés.
Le groupe s'est réuni au printemps 2013 à Tlemcen (Algérie) et se réunira en mars 2014 à Sousse (Tunisie). Il faut noter l'implication des étudiants dans ce groupe d'action ${ }^{[3]}$.

\section{Le groupe d'action prioritaire " recherche "}

Le groupe d'action « recherche » a proposé de mettre en place, dans les congrès d'éducation médicale, des ateliers d'initiation et de perfectionnement aux méthodologies de recherche en éducation médicale. Il est également impliqué dans l'expertise des communications scientifiques soumises lors de ces manifestations et dans la modération de sessions dédiées à la recherche. Il est par ailleurs à l'origine de plusieurs articles publiés dans la revue Pédagogie Médicale $^{[4]}$ et de la traduction du guide de l'AMEE «La boussole de la recherche : une introduction à la recherche en éducation médicale », publié dans la revue en février $2013^{[5]}$. Il a pour prochain objectif de produire des articles et des guides méthodologiques autour de la recherche en éducation médicale, destinés à être publiés dans cette même revue.

\section{Le groupe d'action prioritaire " communication dans la pratique professionnelle en santé "}

Le groupe d'action «communication » s'implique dans les facultés et propose des ateliers sur les 
compétences communicationnelles ${ }^{[6]}$. Suite au dynamisme de nos collègues de Montréal, un colloque de deux jours a été organisé en octobre, en marge du congrès anglophone. Signalons enfin la parution au cours de l'automne 2013 de la deuxième édition de l'ouvrage «La communication professionnelle en santé » (édition ERPI), coordonné par les animateurs de ce groupe.

\section{Trois activités nouvelles ont été proposées et mises sur pied}

La première concerne la simulation. Pour rappel, la simulation "correspond à l'utilisation d'un matériel (comme un mannequin ou un simulateur procédural), de la réalité virtuelle ou d'un patient standardisé pour reproduire des situations ou des environnements de soin, dans le but d'enseigner des procédures diagnostiques et thérapeutiques et de répéter des processus, des concepts médicaux ou des prises de décision par un professionnel de santé ou une équipe de professionnels " ${ }^{[7]}$. Nul doute que cette nouvelle approche (qui en fait n'est pas si nouvelle puisque des simulations avec des poupées en chiffon étaient déjà utilisées au siècle passé) est appelée à se développer, tant pour la formation continue que pour la formation initiale. De nombreux membres de la SIFEM sont engagés dans le développement de centres ou d'activités de simulation. Cela représente un marché nouveau dans lequel certaines firmes se sont engouffrées et l'offre de matériel est énorme. Il est important que le côté technique ne devienne pas prédominant et qu'au-delà de l'apprentissage de gestes, de procédures techniques ou de l'utilisation d'un matériel très sophistiqué, la simulation s'intègre dans une démarche pluridisciplinaire et cohérente par rapport aux objectifs de formation et aux compétences attendues. En particulier, il est indispensable de coordonner les efforts en vue d'harmoniser les innovations dans le cadre du recours aux technologies de l'information, de la communication et de la simulation, au sein de programmes de formation, en particulier en formation initiale. C'est ce souci pédagogique que le groupe formé développera et promouvra, surtout dans le cadre de la formation initiale.
La seconde est la mise sur pied d'un comité éthique de la recherche en pédagogie des sciences de la santé. Ce comité répond à un besoin spécifique. $\mathrm{La}$ recherche en éducation des sciences de la santé partage avec la recherche clinique, dont sont familiers les professionnels de la santé, des questions éthiques liées notamment à la nécessité de garantir la rigueur scientifique du protocole de recherche, de prévenir ou de rendre transparents les conflits d'intérêt potentiels et de prendre en compte les conséquences possibles pour le patient des situations d'enseignement et d'apprentissage qui l'impliquent. Elle s'en distingue néanmoins par des modalités souvent plus éclectiques de construction de la preuve, qui peuvent relever autant d'une démarche de validation de processus que d'une démonstration expérimentale. A cet égard, la recherche en éducation des sciences de la santé est plus proche, sur les plans méthodologique et épistémologique, de la recherche en sciences humaines et sociales. Les objectifs de ce groupe peuvent se résumer comme suit : a) faire le relevé de ce qui existe spécifiquement ou de ce qui peut alimenter la réflexion dans le domaine ; b) élaborer ou préciser des balises fondamentales pour la recherche et les publications et les mettre à disposition de la communauté des chercheurs dans la revue et c) évaluer sur demande les projets de recherche en éducation des sciences de la santé et émettre à leur égard des avis "d'approbation éthique », exigence formelle désormais formulée par les comités de rédaction des revues internationales lors de la soumission des projets d'articles.

La troisième initiative concerne la valorisation des activités d'enseignement. Le déséquilibre entre les poids respectifs des missions de recherche et des missions d'enseignement lors de l'évaluation des dossiers académiques des personnes sollicitant une nomination, une confirmation ou une promotion n'est pas une nouveauté. Sans entrer dans une analyse fine des raisons de ce déséquilibre, nous pouvons avancer que l'absence de structure de présentation, d'évaluation et de valorisation de ces activités d'enseignement, tel que pourrait en constituer un portail internet dédié et administré par un comité de lecture, est pénalisante. Il a donc été proposé qu'un petit groupe de la SIFEM analyse la littérature et ce qui existe dans le domaine 
et fasse des propositions, probablement en 2015 lors du prochain congrès de la SIFEM.

Evoquons enfin le site internet de notre société. Il doit être rénové et adapté aux outils actuellement disponibles pour faciliter le travail collaboratif des groupes de travail de la SIFEM et pour donner aux membres de notre association des informations pertinentes et actualisées; nous négocierons ce virage dans les prochains mois.

\section{Conclusion}

Comme vous le voyez, la SIFEM est avant tout un lieu d'échange et de réflexion prospective sur des problèmes fondamentaux de la pédagogie médicale, pris au sens large, c'est-à-dire impliquant des dimensions stratégiques et institutionnelles. Vous qui lisez cetéditorial et qui êtes intéressé(e) par ce type de problème, rejoignez-nous en adhérant à notre société. De cette manière vous contribuerez aux progrès de la pédagogie et par là, à une meilleure formation des médecins et autres professionnels de la santé.

Soyez les bienvenus...

Jean-François DENEF Président f.f. de la SIFEM mailto : jean-francois.denef@uclouvain.be

Dominique MAILLARD Secrétaire générale de la SIFEM mailto : dominique.maillard@club-internet.fr

\section{Références}

1. Patricio M, Denef J-F. Bienvenue à Lyon en août 2012 ! Participez à la réunion conjointe de l'Association for Medical Education in Europe et de la Société internationale francophone d'éducation médicale. Pédagogie Médicale 2012;13:5-8.

2. Collégial. Consensus mondial sur la responsabilité sociale des facultés de médecine. Pédagogie Médicale 2011;12:37-48.

3. Tudrej BV. La responsabilité sociale des facultés de médecine, un moyen de réconcilier les étudiants avec leur engagement médical. Pédagogie Médicale 2013; 14:73-4.

4. Dory V, Pelaccia T. Une nouvelle initiative de la SIFEM : des ressources pour s'orienter dans le dédale de la recherche en éducation médicale. Pédagogie Médicale 2013;14:5-7.

5. Ringsted C, Hodges B, Scherpbier A. « La boussole de la recherche »: Une introduction à la recherche en éducation médicale : Guide AMEE ${ }^{\circ}$ 56. Pédagogie Médicale 2013;14:49-72.

6. Richard C, Lussier M-T, Galarneau S, Jamoulle O. Compétence en communication professionnelle en santé. Pédagogie Médicale 2010;11:255-72.

7. Granry J-C, Moll MC. Rapport de mission : État de l'art (national et international) en matière de pratiques de simulation dans le domaine de la santé dans le cadre du développement professionnel continu (DPC) et de la prévention des risques associés aux soins : 2012 [On-line] Disponible sur : http:// www.has-sante.fr/portail/plugins/ModuleXitiKLEE/types/FileDocument/doXiti.jsp?id=c_1173128. 